\title{
Maintained goal-directed control with overtraining on ratio schedules
}

Eric Garr ${ }^{1}$, Yasmin Padovan-Hernandez ${ }^{2}$, Patricia H. Janak ${ }^{1,2}, \&$ Andrew R. Delamater ${ }^{3,4}$

${ }^{1}$ Department of Psychological and Brain Sciences, Johns Hopkins University ${ }^{2}$ Solomon H. Snyder Department of Neuroscience, Johns Hopkins University ${ }^{3}$ Department of Psychology, Brooklyn College, City University of New York ${ }^{4}$ Department of Psychology, Graduate Center, City University of New York 


\begin{abstract}
It is thought that goal-directed control of actions weakens or becomes masked by habits over time. We tested the opposing hypothesis that goal-directed control becomes stronger over time, and that this growth is modulated by the overall action-outcome contiguity. Despite group differences in action-outcome contiguity early in training, rats trained under random and fixed ratio schedules showed equivalent goal-directed control of lever pressing that appeared to grow over time. We confirmed that goal-directed control was maintained after extended training under another type of ratio schedule - continuous reinforcement - using specific satiety and taste aversion devaluation methods. These results add to the growing literature showing that extensive training does not reliably weaken goal-directed control and that it may strengthen it, or at least maintain it.
\end{abstract}


Flexible behavior requires an understanding of how one's actions influence the environment around them. Goal-directed control, defined as control over an action by its anticipated consequence, is known to be influenced by the schedule of reinforcement that the action has been acquired under. Goal-directed control has been shown to emerge under random ratio schedules more readily than random interval schedules (Gremel \& Costa, 2013), and more readily under fixed interval than random interval schedules (DeRusso et al., 2010; Garr et al., 2020). In a recent report (Garr et al., 2020), it was argued that a potentially important variable driving these schedule effects is the overall action-outcome (A-O) contiguity, defined as the mean time between an instrumental action and its subsequent outcome. Specifically, the hypothesis maintains that (1) goal-directed control of instrumental actions grows with repeated experience of A-O pairings, and (2) the overall A-O contiguity controls the rate of this growth, such that shorter times between actions and outcome facilitate the onset of goal-directed control while longer times have a retarding effect. The idea that learning becomes stronger with more favorable contiguity conditions is an old idea with significant empirical support (e.g. Balsam, Drew, \& Gallistel, 2010).

The idea that goal-directed control becomes stronger, or is at least maintained, with learning receives some support in the literature (Colwill \& Rescorla, 1985; 1988; Colwill \& Triola, 2002; Garr et al., 2020; Jonkman et al., 2010) but contradicts the popular idea that goal-directed control becomes weaker or masked by habitual control over training (Adams, 1982; Smith \& Graybiel, 2013; Tricomi et al., 2009). Furthermore, the idea that A-O contiguity is a primary determinant of goal-directed control needs further testing. Urculay and Jonkman (2019) trained rats on a continuous reinforcement (CRF) schedule but varied A-O contiguity between groups. They observed that rats trained with a $20 \mathrm{~s}$ delay between lever press and food reward failed to develop goal-directed control, whereas rats trained with immediate reward were goal-directed. We sought to follow up on these ideas by training rats on random and fixed ratio schedules and subsequently testing goal-directed control in tests of reward devaluation. We predicted that the random schedule would induce relatively poor A-O contiguity especially early in training compared to the fixed schedule, and this should translate to slower growth of goal-directed control. We also predicted that the magnitude of goal-directed control would grow over the course of training rather than weaken.

Forty-Eight Long-Evans rats (24 male, 24 female) were run in three replications ( $n=16$ per replication). They were maintained at $85 \%$ of their ad libitum weight. Rats were first given one session of magazine training with one pellet type (TestDiet MLabRodent $45 \mathrm{mg}$ grain or Bioserv $45 \mathrm{mg}$ purified). Twenty pellets were delivered on a $60 \mathrm{~s}$ random time schedule. Rats were then trained to press a lever on a CRF schedule such that each lever press yielded one pellet. Following one session of CRF, the reinforcement schedule was switched to either a fixed ( $n=$ $24)$ or random $(n=24)$ ratio schedule. The fixed schedule was arranged so that a pellet was delivered into the magazine every $20(\mathrm{FR}-20 ; n=16)$ or $25(\mathrm{FR}-25 ; n=8)$ presses. The random schedule was arranged so that, each time the lever was pressed, a pellet was delivered with a 0.05 (RR-20; $n=16$ ) or 0.04 (RR-25; $n=8$ ) probability. Two rats (one FR and one RR) did not learn to lever press and were excluded. At the beginning of each session, the lever was inserted and remained available until 50 pellets were earned or 60 min elapsed, whichever occurred first. Ratio training continued for 30 daily sessions, with devaluation test cycles conducted repeatedly 
after sessions 2,10,20, and 30. These test intervals were chosen based on a previous study with interval schedules (Garr et al., 2020), with an additional test cycle to examine truly extensive training.

Each devaluation test cycle was comprised of two tests separated by a session of retraining. Prior to the first test, rats were isolated in wire cages and given one hour of unlimited access to either the pellet type that was associated with lever pressing ("devalued" test) or the other pellet used as a control for general satiety ("valued" test). Beginning two days prior to the first test, rats were pre-exposed to the unfamiliar pellet type and consumed 20 pellets each day after training sessions. Immediately after the satiation period, rats were placed in the operant chambers and given a 5-min test in which the lever was available but no rewards were delivered. The retraining session was run the following day and was identical to ratio training. The next day, each rat was sated on the other pellet type and tested again. Group assignment, pellet outcome, sex, and order of devaluation tests were counterbalanced.

All statistical tests were conducted using analysis of variance (ANOVA) with $\alpha=.05$.

Significant interactions were followed up with one-way ANOVAs and mutually orthogonal post hoc contrasts using the recommendations of Rodger (1974). Degrees of freedom for follow-up one-way ANOVAs were derived from Satterthwaite's (1946) approximation. We also provide a measure of effect size based on Perlman and Rasmussen's (1975) estimate of the noncentrality parameter $\Delta$.

During instrumental training, rats showed increases in lever pressing rates across sessions (Fig 1A). An ANOVA with session, schedule randomness, and ratio value as factors showed only a main effect of session $(F(3,126)=211.94, p<.05)$. We recorded the time from each lever press to the subsequent pellet delivery and computed an average A-O temporal distance per session (Fig 1B). An ANOVA on this measure revealed significant main effects of schedule randomness $(F(1,44)=15.49, p<.05)$ and session $(F(1,44)=49.19, p<.05)$, and a randomness $\mathrm{x}$ session interaction $(F(1,44)=7.97, p<.05)$. Follow-up one-way ANOVAs on sessions 2, 10, 20, and 30 revealed a significant difference only during session $2(F(1,176)=32.64, \Delta=31.27, p<.05)$, with the RR group showing weaker A-O contiguity compared to the FR group.

\section{[Insert Figure 1 here]}

For reward devaluation tests, we subjected mean lever pressing rates to an ANOVA with session, outcome value, schedule randomness, ratio value, and sex as factors. Pressing rates are shown in Fig 2A. Significant main effects were detected only for session $(F(3,114)=16.47, p<.05)$, outcome value $(F(1,38)=87.37, p<.05)$, and ratio value $(F(1,38)=10.80, p<.05)$. The main effect of ratio value reflects the fact that the FR-25 and RR-25 subgroups pressed at overall higher rates than the FR-20 and RR-20 subgroups, but since this variable did not interact with any other variable, we chose to collapse across these subgroups. The only significant interaction was between session and outcome value $(F(3,114)=8.61, p<.05)$. A follow-up one-way ANOVA collapsed across groups revealed significant differences among the eight tests $(F(7,308)$ $=26.86, \Delta=176.74, p<.05)$. Post hoc contrasts could not detect a significant devaluation effect after 2 days of ratio training, but showed robust devaluation effects after 10, 20, and 30 days $\left(F^{\prime} s(7,308)>4.72, \mathrm{p}<.05\right)$ as well as a greater overall rate of pressing during the final three test 
cycles compared to the first $(F(7,308)=6.41, \mathrm{p}<.05)$. We also calculated devaluation scores as (valued - devalued)/(valued + devalued) and plotted individual scores (Fig 2B). These analyses suggest, for both groups, that, once manifested, goal-directed control was maintained over training.

\section{[Insert Figure 2 here]}

We also measured consumption during the satiation periods (Fig 2C). There was a main effect of session $(F(3,132)=26.70, p<.05)$, but no main effect of value or group $\left(F^{\prime} s(1,44)<.73, p^{\prime}\right.$ s $>$ $.05)$. There were no interactions. The main effect of session reflects the fact that consumption increased over tests. Consumption scores were calculated as (devalued - valued)/(devalued + valued) and plotted for individual rats (Fig 2D). We conclude that goal-directed control of instrumental actions is initially difficult to detect but, once detected, is maintained comparably on fixed and random ratio schedules, and seems unaffected by variations in A-O contiguity early in training.

Our hypothesis that differences in A-O contiguity between FR and RR groups should have resulted in different acquisition rates of goal-directed control was not supported by the data, although it is worth noting that A-O contiguity differed between groups only early in training and goal-directed control was detectable in both groups sometime between sessions 2 and 10 . Further tests between these two training levels would be required to more fully evaluate the hypothesis. The data also support the view that goal-directed control may strengthen with overtraining, and do not match the assumption that actions transition to outcome-insensitive habits with overtraining on ratio schedules. Therefore, we next sought to replicate the first experiment that reported habit formation with overtraining on a ratio schedule (Adams, 1982). This experiment involves training separate groups of rats for either 2 or 10 sessions of CRF followed by testing after reward devaluation. In the original report, rats given 2 training sessions showed goal-directed control of lever pressing, but goal-directed control was attenuated in rats given 10 training sessions. That experiment employed taste aversion to devalue the instrumental outcome. We continued with our method of using specific satiety but, in a separate experiment, also used taste aversion with lithium chloride $(\mathrm{LiCl})$ because it has been suggested that the specific satiety procedure may break habits (Bouton et al., 2020).

In the specific satiety experiment, sixteen rats ( 8 male, 8 female) were treated similarly as before but with the following exceptions. Following magazine training, rats were trained only on CRF schedules for either $2(n=8)$ or $10(n=8)$ sessions. A single devaluation test cycle (5 min. tests) was conducted after the assigned length of training. Satiation occurred in the home cage. The pellet types used were Bio-serv $45 \mathrm{mg}$ grain pellets and Bio-serv $45 \mathrm{mg}$ banana flavored sucrose pellets. One rat in each group did not learn to lever press and were excluded.

The degree of goal-directed control appeared to differ between groups (Fig 3A), and this was supported by statistical analysis. A session $\mathrm{x}$ outcome value $\mathrm{x}$ sex ANOVA revealed a main effect of outcome value $(F(1,12)=31.78, p<.05)$ and an outcome value $\mathrm{x}$ group interaction $(F(1,12)=8.59, p<.05)$. No other main effects or interactions reached significance. Follow-up one-way ANOVAs did not detect a devaluation effect in the 2-day group $(F(1,12)=3.66, p>$ $.05)$ but did detect an effect in the 10-day group $(F(1,12)=36.71, \Delta=29.59, p<.05)$. 
Devaluation scores for individual rats showed a notable difference in variability between groups - while there was a high degree of variability within the 2-day group, all the rats within the 10-day group showed a strong devaluation effect (Fig 3B). We also measured consumption during the satiation periods (Fig 3C). There was no main effect of outcome value $(F(1,12)=$ $2.58, p>.05)$, a main effect of group $(F(1,12)=7.68, p<.05)$, and no outcome value $\mathrm{x}$ group interaction $(F(1,12)=.90, p>.05)$. Consumption scores are plotted for individual rats (Fig 3D). Of note, the outlier in Fig 3B is not the same rat as the outlier in Fig 3D. This analysis supports the conclusion that goal-directed control was greater after 10 vs. 2 days of CRF training.

\section{[Insert Figure 3 here]}

In the $\mathrm{LiCl}$ experiment, thirty-two rats (15 male, 17 female) were evenly divided into a 2-day and a 10-day training group. Rats were once again trained to press a lever on a CRF schedule for either grain or banana sucrose pellets. Pellet outcome, group assignment, and sex were counterbalanced. Reward devaluation was conducted over 5 two-day cycles. During the first day of each cycle, all rats were given 50 pellets in the absence of the lever in the operant box randomly over 15 mins followed by an injection of either $\mathrm{LiCl}$ for the devalued subgroups $(0.3$ $\mathrm{M}, 15 \mathrm{ml} / \mathrm{kg}$ ) or saline for the valued subgroups. During the second day, rats were placed in the operant box without access to pellets for 15 mins and then given an injection of either saline for the devalued subgroups or $\mathrm{LiCl}$ for the valued subgroups.

During the final training session, the mean presses per min for the 2-day valued, 2-day devalued, 10-day valued, and 10-day devalued groups were 5.02, 5.03, 12.35, and 11.51, respectively. One rat from the 10-day devalued group failed to learn to press and was excluded. Taste aversion proceeded smoothly (Fig 3F), with the exception that one rat from the 2-day devalued group did not receive pellets during the final session due to a technical malfunction and was excluded. Pressing rates during the 5 min. tests are presented in Fig 3E. A session $x$ outcome value $x$ sex ANOVA showed a main effect of outcome value $(F(1,22)=6.45, p<.05)$, reflecting the fact that pressing rates were greater for valued than devalued groups regardless of training length. No other main effects or interactions reached significance. This analysis supports the conclusion that goal-directed control was equally strong after 10 vs. 2 days of CRF training.

The present set of experimental results add to the growing literature showing that extensive training does not weaken goal-directed control and can strengthen or maintain it (Colwill \& Rescorla, 1985; 1988; Colwill \& Triola, 2002; Corbit et al., 2012; de Wit et al., 2018; Garr et al., 2020; Garr \& Delamater, 2019; Jonkman et al., 2010; Pool et al., 2021; Thrailkill et al., 2018). Rats trained on fixed and random ratio schedules displayed equivalent levels of goal-directed control that became more evident between sessions 2 and 10 of instrumental training and was maintained from sessions 10 to 30 . This is despite the fact that fixed and random ratio schedules induced a large initial difference in A-O contiguity, which was hypothesized to drive differences in the rate of A-O learning. A-O contiguity may be less critical for A-O learning than originally thought (DeRusso et al., 2010; Garr et al., 2020). However, more direct manipulations of A-O contiguity with a wider variety of operant schedules may be necessary to further evaluate this idea (e.g. Urculay \& Jonkman, 2019). 
It has long been recognized that the schedule of reinforcement influences the degree to which an action becomes goal-directed (Dickinson, 1985), and we can now update our knowledge of these schedule effects. Specifically, after rats or mice have been trained for 6-10 training sessions to press a single lever, lever-pressing is reliably goal-directed under fixed ratio, random ratio, and fixed interval schedules, but not random interval schedules (DeRusso et al., 2010; Dickinson, Nicholas, \& Adams, 1983; Garr et al., 2020; Gremel \& Costa, 2013). What we have shown in our current and previous (Garr et al., 2020) reports is that once instrumental training reaches 20 sessions, lever-pressing becomes goal-directed under all schedules. It is notable that extensive training is often defined in a range of 6 to 14 sessions (e.g. Malvaez et al., 2018; Colwill \& Rescorla, 1988) and training rarely exceeds that limit.

One caveat in interpreting the results of the current set of experiments is that lower overall levels of pressing after two training sessions potentially impede the ability to detect a devaluation effect because pressing rates are close to the floor. Strong devaluation effects are more easily detectable when baseline levels are higher. Moreover, across studies, consumption during the satiation periods increased over time. This could reflect increasing hunger, which could raise pressing rates higher above the floor and thus facilitate the detection of a devaluation effect for rats that underwent extensive training. It is therefore difficult to state with certainty that goaldirected control grew between session 2 to session 10, only that it did not decline. However, it is worth pointing out that even when these confounds are controlled for, there is still strong evidence for strengthening of goal-directed control with extensive training (Colwill \& Rescorla, 1988).

An interesting question is why random interval schedules produce sluggish goal-directed learning. A recent study in humans found that, after training on a random interval schedule, selfreported stress levels served as a moderating variable of performance on a specific satiety devaluation test such that high stress levels predicted devaluation-insensitive performance (Pool et al., 2021). In a randomized control study, a stress manipulation also predicted devaluationinsensitive performance (Schwabe \& Wolf, 2009), and stress has been appealed to as part of a neurobiological mechanism mediating the effect of a high-fat diet on instrumental sensitivity to food devaluation (Tantot et al., 2017). Whether random interval schedules induce more stress than fixed interval or ratio schedules in rodents, and whether this can account for devaluation effects under specific satiety and/or taste aversion paradigms, remains to be investigated.

Of note, we found that goal-directed control either grew or was maintained when rats were tested after 10 vs. 2 sessions on a CRF schedule, and this was confirmed with two types of reward devaluation methods. This result constitutes a failure to replicate the first study that examined the effect of training length on instrumental sensitivity to reward devaluation (Adams, 1982). It is difficult to decipher why the results did not replicate. One possibility is a difference in reward types. Adams (1982) used sucrose pellets while we used banana-flavored sucrose pellets and grain pellets, and there is some evidence that the reward type can change performance during devaluation tests (Vandaele, Pribut, \& Janak, 2017). Nonetheless, it is noteworthy that there are not many instances where the effects of overtraining on ratio schedules have been examined in relation to goal-directed control. To our knowledge, actions appear to convert to outcomeinsensitive "habits" on only a rather limited set of training conditions - namely, training on fairly lean random interval schedules. This leads us to question the generality of the phenomenon. 


\section{Acknowledgements}

The research reported here was supported by a NIGMS grant (SC1 DA034995) awarded to ARD and a NIDA grant (R01 DA035943) award to PHJ. We thank Badrunnesa Bushra, Chloé PierreLouis, Norman Tu, and Dan Siegel for assistance with data collection. Correspondence

concerning this article should be addressed to Eric Garr, Department of Psychological and Brain Sciences, Johns Hopkins University, 3400 North Charles Street, Baltimore, MD, 21218. E-mail: egarr1@jh.edu 


\section{References}

Adams, C. D. (1982). Variations in the sensitivity of instrumental responding to reinforcer devaluation. The Quarterly Journal of Experimental Psychology Section B: Comparative and Physiological Psychology, 34(2), 77-98. https://doi.org/10.1080/14640748208400878

Balsam, P. D, Drew, M. R., \& Gallistel, C. R. (2010). Time and Associative Learning. Comparative Cognition \& Behavior Reviews, 5, 1-22.

https://doi.org/10.3819/ccbr.2010.50001

Bouton, M. E., Broomer, M. C., Rey, C. N., \& Thrailkill, E. A. (2020). Unexpected food outcomes can return a habit to goal-directed action. Neurobiology of Learning and Memory, 169, 107163.

Colwill, R. M., \& Rescorla, R. a. (1985). Instrumental responding remains sensitive to reinforcer devaluation after extensive training. Journal of Experimental Psychology: Animal Behavior Processes, 11(4), 520-536.

Colwill, R. M., \& Rescorla, R. A. (1988). The role of response-reinforcer associations increases throughout extended instrumental training. Animal Learning \& Behavior, 16(1), 105-111.

Colwill, R. M., \& Triola, S. M. (2002). Instrumental responding remains under the control of the consequent outcome after extended training. Behavioural Processes, 57(1), 51-64

Corbit, L. H., Nie, H., \& Janak, P. H. (2012). Habitual alcohol seeking: Time course and the contribution of subregions of the dorsal striatum. Biological Psychiatry, 72(5), 389-395. https://doi.org/10.1016/j.biopsych.2012.02.024

de Wit, S., Kindt, M., Knot, S. L., Verhoeven, A. A. C., Robbins, T. W., Gasull-camos, J., \& Gillan, C. M. (2018). Shifting the Balance Between Goals and Habits: Five Failures in Experimental Habit Induction. Journal of Experimental Psychology: General, 147(7), 1043-1065. https://doi.org/10.1037/xge0000402.supp

DeRusso, A. L., Fan, D., Gupta, J., Shelest, O., Costa, R. M., \& Yin, H. H. (2010). Instrumental uncertainty as a determinant of behavior under interval schedules of reinforcement. Frontiers in Integrative Neuroscience, 4(May), 1-8. https://doi.org/10.3389/fnint.2010.00017

Dickinson, A. (1985). Actions and Habits: The Development of Behavioural Autonomy. Philosophical Transactions of the Royal Society of London. Series B, Biological Sciences, 308(1135), 67-78.

Dickinson, A., Nicholas, D. J., \& Adams, C. D. (1983). The effect of the instrumental training contingency on susceptibility to reinforcer devaluation. The Quarterly Journal of Experimental Psychology Section B, 35(1), 35-51. https://doi.org/10.1080/14640748308400912 
Garr, E., Bushra, B., Tu, N., \& Delamater, A. R. (2019). Goal-Directed Control on Interval Schedules Does Not Depend on the Action-Outcome Correlation. Journal of Experimental Psychology: Animal Learning and Cognition, 46(1), 47-64. https://doi.org/10.1037/xan0000229

Garr, E., \& Delamater, A. R. (2019). Exploring the relationship between actions, habits, and automaticity in an action sequence task. Learning and Memory, 26(4), 128-132. https://doi.org/10.1101/lm.048645.118

Gremel, C. M., \& Costa, R. M. (2013). Orbitofrontal and striatal circuits dynamically encode the shift between goal-directed and habitual actions. Nature Communications, 4(May), 1-12. https://doi.org/10.1038/ncomms3264

Jonkman, S., Kosaki, Y., Everitt, B. J., \& Dickinson, A. (2010). The role of contextual conditioning in the effect of reinforcer devaluation on instrumental performance by rats. Behavioural Processes, 83(3), 276-281. https://doi.org/10.1016/j.beproc.2009.12.017

Malvaez, M., Green, V. Y., Matheos, D. P., Angelillis, N. A., Murphy, M. D., Kennedy, P. J., ... Wassum, K. M. (2018). Habits Are Negatively Regulated by Histone Deacetylase 3 in the Dorsal Striatum. Biological Psychiatry, 84, 383-392. https://doi.org/10.1016/j.biopsych.2018.01.025

Pool, E. R., Gera, R., Fransen, A., Perez, O. D., Cremer, A., Aleksic, M., ... O’Doherty, J. P. (2021). Determining the effects of training duration on the behavioral expression of habitual control in humans: a multi-laboratory investigation. PsyArXiv.

Rodger, R. S. (1974). Multiple contrasts, factors, error rate and power. British Journal of Mathematical and Statistical Psychology, 27, 179-198.

Satterthwaite, F. E. (1946). An approximate distribution of estimates of variance components, Biometrics Bulletin, 2, 110-114. http://www.jstor .org/stable/3002019

Schwabe, L., \& Wolf, O. T. (2009). Stress prompts habit behavior in humans. Journal of Neuroscience, 29(22), 7191-7198. https://doi.org/10.1523/JNEUROSCI.0979-09.2009

Smith, K. S., \& Graybiel, A. M. (2013). A dual operator view of habitual behavior reflecting cortical and striatal dynamics. Neuron, 79, 361-374. https://doi.org/10.1016/j.neuron.2013.05.038

Tantot, F., Parkes, S. L., Marchand, A. R., Boitard, C., Naneix, F., Layé, S., ... Ferreira, G. (2017). The effect of high-fat diet consumption on appetitive instrumental behavior in rats. Appetite, 108, 203-211. https://doi.org/10.1016/j.appet.2016.10.001

Thrailkill, E. A., Trask, S., Vidal, P., Alcalá, J. A., \& Bouton, M. E. (2018). Stimulus control of actions and habits: A role for reinforcer predictability and attention in the development of 
habitual behavior. Journal of Experimental Psychology: Animal Learning and Cognition, 44(4), 370-384. https://doi.org/10.1037/xan0000188

Tricomi, E., Balleine, B. W., \& O'Doherty, J. P. (2009). A specific role for posterior dorsolateral striatum in human habit learning. European Journal of Neuroscience, 29(11), 2225-2232. https://doi.org/10.1111/j.1460-9568.2009.06796.x

Urcelay, G. P., \& Jonkman, S. (2019). Delayed rewards facilitate habit formation. Journal of Experimental Psychology: Animal Learning and Cognition, 45(4), 413-421.

Vandaele, Y., Pribut, H. J., \& Janak, P. H. (2017). Lever Insertion as a Salient Stimulus Promoting Insensitivity to Outcome Devaluation. Frontiers in Integrative Neuroscience, 11(September), 1-13. https://doi.org/10.3389/fnint.2017.00023 

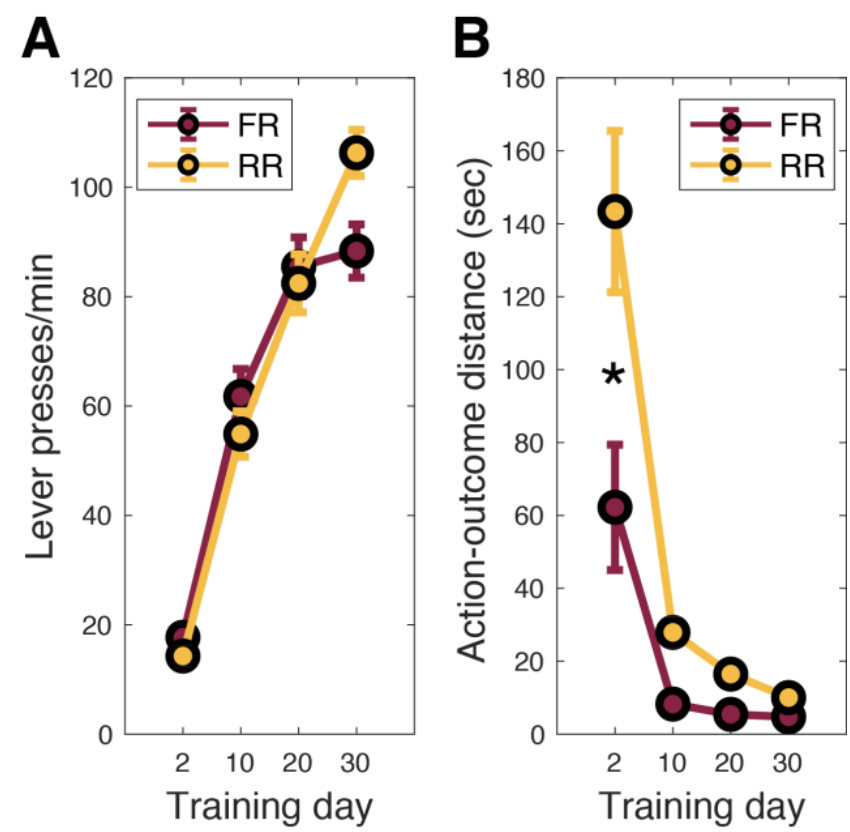

Figure 1. (A) Mean lever presses per minute across training sessions. (B) Mean time separating an individual lever press from the subsequent pellet delivery across sessions. Data are collapsed across FR/RR-20 and FR/RR-25 subgroups. Vertical bars $=$ SEM. $*=p<.05$ 

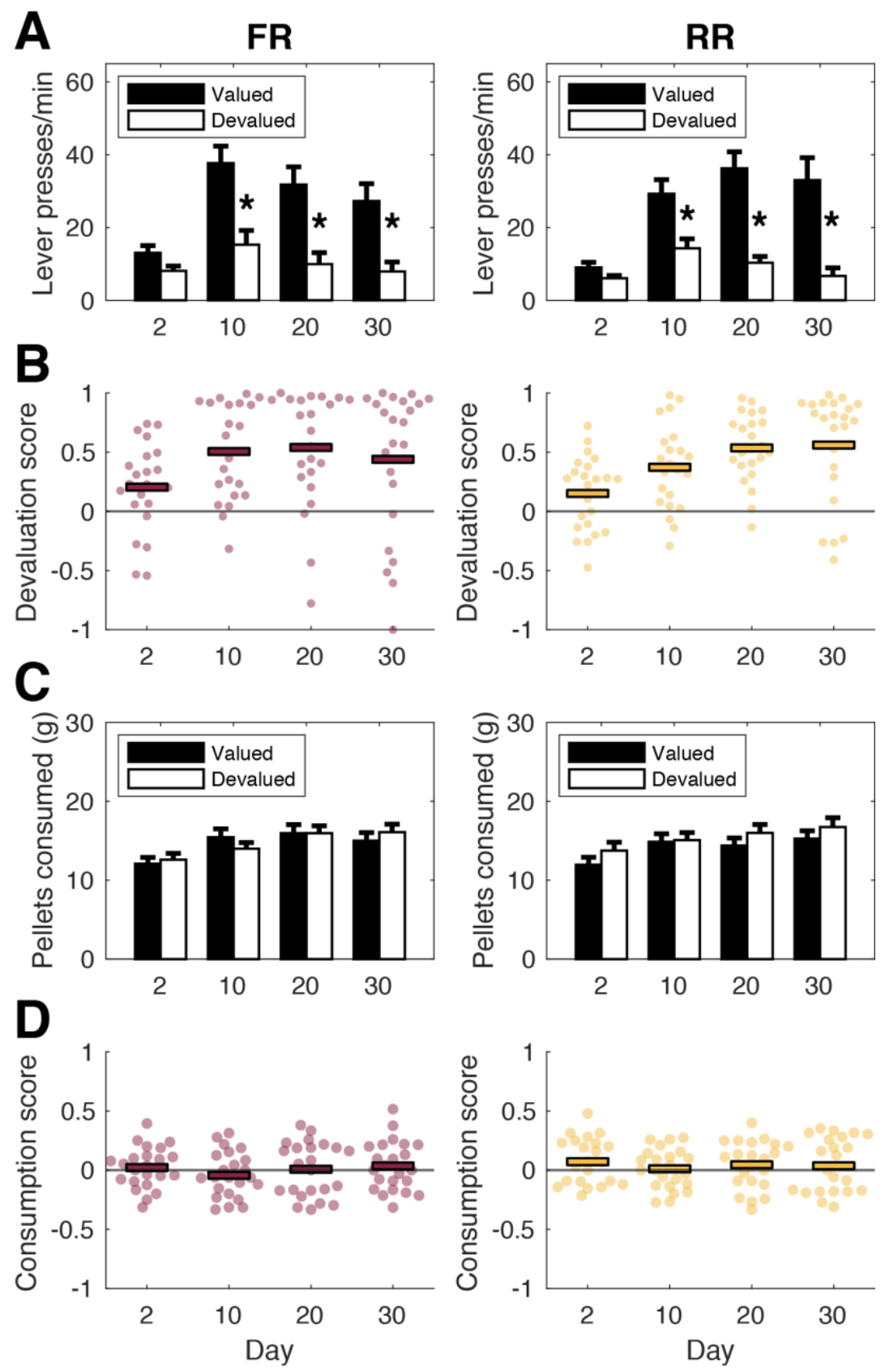

Figure 2. (A) Lever pressing rates during non-rewarded tests as a function of test type (valued vs. devalued) and training length. Left: fixed ratio group. Right: random ratio group. Rats were repeatedly tested. $*=p<.05$ (B) Devaluation scores over time. Scores were calculated as (valued - devalued)/(valued + devalued). Circles are individual rats and horizontal bars are means. (C) Amount of pellets consumed during 1 hour satiation periods prior to testing as a function of test type (valued vs. devalued) and training length. (D) Consumption scores over time. Scores were calculated as (devalued - valued)/(valued + devalued). 

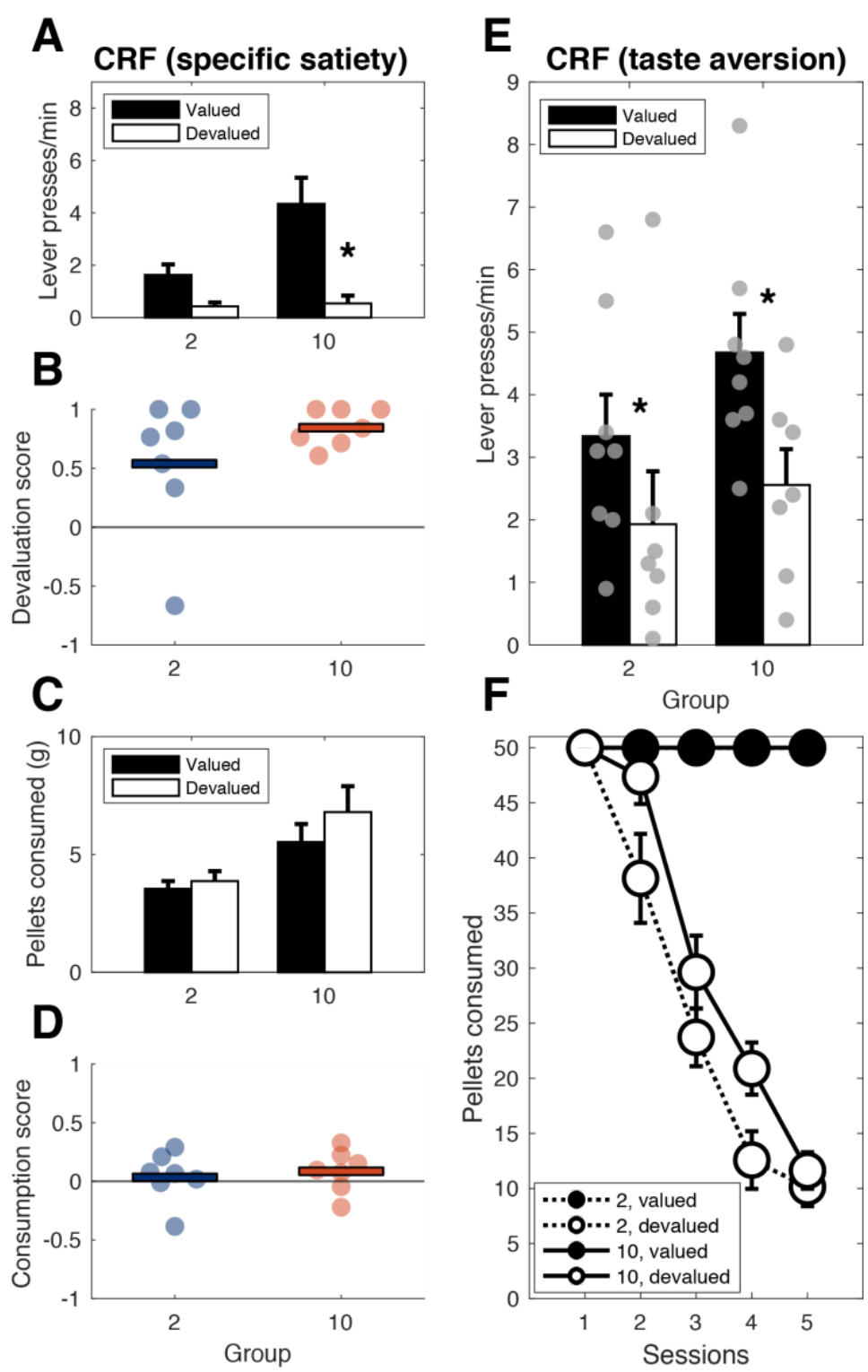

Figure 3. (A) Lever pressing rates during non-rewarded tests as a function of test type (valued vs. devalued) and group following either 2 or 10 sessions of CRF. Devaluation tests were conducted using specific satiety. ${ }^{*}=p<.05$ (B) Devaluation scores calculated as (valued devalued)/(valued + devalued). Circles are individual rats and horizontal bars are means. (C) Amount of pellets consumed during 1 hour satiation periods prior to testing as a function of test type (valued vs. devalued) and group. (D) Consumption scores were calculated as (devalued valued)/(valued + devalued). (E) Lever pressing rates during non-rewarded tests as a function of test type (valued vs. devalued) and group following either 2 or 10 sessions of CRF. Devaluation tests were conducted using taste aversion. (F) Pellets consumed over time during taste aversion. 